\title{
The Promise of Fuzzy Logic in Generalised Music Composition
}

\author{
Wendy Suiter \\ University of Wollongong, \\ NSW, Australia \\ ws999@uow. edu.au
}

\begin{abstract}
The paper outlines the rationale for using Fuzzy Logic, and Granular Computing, to emulate compositional decision making processes. Significant features of this Fuzzy Logic framework are that ambiguity in the music is maintained, while allowing the evolution of unfolding processes which reflects the temporal nature of music as performed. Granular Computing and Fuzzy Logic have been designed for physical and IT engineering applications to automate complex tasks. Fuzzy Logic is not only useful as an analytical concept, but also, can be generally applied to the production of music itself through a Fuzzy Logic control system. As artificial intelligence design and computing power both improve, it may well become possible to perform this work by digital means. My present research will contribute to this development through the identification of the significant compositional elements and their connective grammar. Once, these have been determined, then automated ways to compose music can be developed.
\end{abstract}

Keywords: Fuzzy Logic, Granular Computing, Music Composition, Algorithmic Music.

\section{Introduction}

If you were composing a piece of music from scratch, how would you decide what to do? Naturally, you would first consider the general requirements for the piece. Of course there are not only technical constraints, like how long is the piece to be, and what technology is available for the performance, but there are also contextual considerations such as the location of, and the reason for, the performance.

Yet once these initial conditions have been clarified, there are many more decisions waiting to be made. What about the first sound event: its pitch? It's duration? It's amplitude? What about the next sound event: its pitch? It's duration? It's amplitude? What about every other event that falls between this and the final one? How would you decide what to do?

Composers are faced with making these decisions every time they work. To reduce the number of micro-level decisions needed, my goal is to find a set of elements and rules, which will ultimately enable me to construct an algorithmic compositional system which can produce expressive music if so desired. Typical analysis of conventional music throws little light on these questions [13]. 
My present goal is to produce a theoretical model of music that is applicable across all genres of music, including both acoustic music and computer music. This is substantially different from other theories which, typically, can only be applied to one style of music. For instance, Forte's work applying classical set theory to music analysis is limited to atonal music [6]. He specifically states that his work is not applicable to serial music, which, interestingly, is the more algorithmic compositional technique of the two.

Fuzzy Logic principles seem to be the most useful way of describing completely, both the elements, and their interactions, which contribute to the aesthetic outcomes of music. This is supported by the application of Efstathiou's decision making paradigm [4] to the research itself, which will be discussed in more detail later in the paper.

\section{Literature Review}

Very little work has been done in the application of Fuzzy Logic to music composition, although there are several papers which outline the need for this work. Two composers have mentioned the conceptual incorporation of Fuzzy Logic in automated musical decision making, without any justification for the specific inclusion of FL as a methodology in the models. Yet several others have developed specific musical applications such as chord generators, but they have not engaged with modelling complete systems from an aesthetic perspective.

In the first of the two papers mention the conceptual use of Fuzzy Logic in music composition is Whalley's paper presented to the 2002 International Computer Music Conference [14]. In this paper Whalley provides a brief overview of literature on emotion as musical narrative, semiotics of music in relation to automated composition systems. This review shows that there is a need for this type of work, without providing any rationale for the potential inclusion of Fuzzy Logic methodology.

Milicevic has published a series of papers exploring the reception of computer music, each reframing the same idea as new philosophical frameworks become available. One of his papers uses some elements of Fuzzy Logic as the means to advance his argument [9]. Milicevic argues that computer music composers need to consider the human brain mechanisms of the audience when composing their music, as this will result in a more positive reception of their music. He proposes the development of an automated Fuzzy Logic system which could analyse each piece of music for its patterning, which would then enable the composer to determine whether the music would engender a positive reception.

Several composers have incorporated Fuzzy Logic into specific creative work. Cadiz produces continuous a-rhythmic compositions through the control of several sound synthesis parameters [2]. That is, the musical work unfolds as the synthesised timbres change. Other programmers have also made modules which work in various programming languages to generate single musical elements using Fuzzy Logic engines. Elsea [5], has developed a chord generating mechanism, while Sorensen and Brown [12] have developed a Fuzzy Logic module for their jMusic program which operates on volume levels. While this work can potentially be developed into complex algorithms for a large range of musical elements, they have not engaged here with the concept of modelling complete musical systems from an aesthetic perspective. 


\section{Efstathiou's Decision Making Paradigm}

Janet Efstathiou's paper on rule-based multi-attribute decision making [4] sets out a decision making process/paradigm that is immediately comprehensible, espouses ethics of including the decision maker(s) in the entire process, and is entirely human oriented. Indeed in simple cases, computers and numerical calculations (whether based on fuzzy logic or not) may not be necessary, because the computation can be done entirely in natural language, “... with pencil, paper and common sense." [4]

There are two parts to Efstathiou's decision-making paradigm. The first part deals with how to find an appropriate methodology to calculate potential solutions for a specific problem given the environment in which the question is being posed. This is done by asking a series of meta-questions [4]. The second part deals with developing a preference ordering for the various proposed outcomes to the specific multi-attribute problem. This takes place by the decision makers ranking each possible solution against the complete range of attributes to be addressed in the solution. The process she suggests is based on Fuzzy Logic using natural language, taking place in an iterative learning environment.

The paradigm seems very similar to Adaptive Resonance Theory which was developed in the AI context of machine learning. As a result, ART is very engineering oriented with much technical writing. As ART has become an accepted form of machine learning for robotics, it would seem that the date of Efstathiou's model is not relevant. Efstathiou [4] recognises the relationship between her model and robotics in her conclusion, making the distinction between machines and human decision making. As Fuzzy ART had not been developed at the time of her article this distinction was important. However, the development of Fuzzy ART [3] [7] has produced a type of machine learning which is very close to Efstathiou's paradigm. Fuzzy ART, typically, seems to be concerned with machine learning and system control of automated processes.

What is essentially useful about Efstathiou's model is its orientation towards human decision making, means that the mental and intuitive requirements of humans are considered at every step.

\subsection{Part 1: Finding the Solution Methodology}

Applying the notion of meta-questions from the first part of Efstathiou's model leads through an iterative process of refining and clarifying the best methodology to use for any specific decision making problem. Her methodological model [4] is based on the proposition that it is not practical for all possible problem solving methods to be tested. The question then becomes one of deciding which methodology will provide the best possible solutions for any given problem.

The iterative process Efstathiou proposes, teases out the essential attributes, in relation to the problem, that the solution method must hold. Thus if the solution method addresses all the significant issues surrounding the original question, then the solution to a specific problem provided by the selected problem solving method will provide a satisfactory and useful answer. The iterative process, when applied to this research question, results in the choice of Granular Computing and Fuzzy Logic. 
The process of establishing a useful methodology is worked through below. In working through the process I have relabelled some of her steps to provide a more clearly defined pathway.

Question (Q0) derived from an underlying research topic: Is it possible to compose process music which is expressive?

Meta Question (MQ1) arises from the original question (Q0) on the basis that it is not possible to test most possible methodologies for their usefulness, how is a good solution method chosen from all the possible methods?

MQ1 is answered here by MA1: The solution must be a method which can become the compositional technique when it is fully developed.

MQ2 follows from MA1 on the basis that given there may be multiple frameworks which can do this, how do I choose which technique to use to resolve this question? MQ2 is answered here by MA2: The solution method must be a clearly defined rule based procedure, that is an algorithmic process, which is logical and transparently meaningful, while reflecting the essential qualities of music itself.

MQ3 follows from MQ2 on the basis that given there may be multiple frameworks which can do this, how do I choose which technique to use to resolve this question? MQ2 is clarified here by MQ3: What will ensure that these essential qualities will be reflected in the solution method?

MQ3 is answered by MA3: The solution must be an algorithmic problem solving model which is capable of handling imprecision and vagueness, while permitting both time and context dependent, multi-attribute decision making, while remaining transparent and inherently meaningful.

MA4 follows from MQ3 on the basis that it is not possible to test most possible solutions, how is a good solution chosen from all the possible solutions? MA4 here is: A good solution method is Granular Computing (derived from principles of fuzzy logic) based on natural language.

\subsection{Part 11: Using the Solution Methodology to Solve the Problem}

The second part of her model is concerned with using the solution finding methodology discovered through the meta-question process to find a range of possible solutions, and a method for assessing the most optimal solution for the given problem. The evaluation method involves obtaining a "preference ordering" of the attributes which must be addressed by the specific solution to this particular problem. This preference ordering is derived through a set of natural language IF/THEN rules.

Each IF/THEN rule compares a single element from each of two attributes by comparing two antecedents to produce a consequent mapping into an 'evaluation space' (my term) which will contain several fuzzy sets such as Poor, OK, Very Good. Each IF/THEN rule results in an individual evaluation outcome for this specific problem. (For example: IF... the dynamic level is 'very soft' ...AND...the pitch is 'very low'...THEN ...this option is POOR.) But the determined level of the consequent assessment will depend on the particular problem-solving context in which the decision is being made, so that in some circumstances, such as when evaluating 'music for relaxation', this rule may be changed to produce a consequent evaluation of VERY GOOD. 
Then the elements of each alternative solution are simultaneously fed through the set of rules to provide the evaluation set for that solution, which, in total, will give the overall quality of that alternative.

Each one of the alternative solutions can then be given a "preference ordering" according to the resulting quality of the evaluation set for that alternative solution.

\section{Fuzzy Logic and the Specific Properties of Music}

The production of musical art involves consideration of its intrinsic qualities. It is Time dependent; an Abstract art form; it includes a combination of emotional and intellectual qualities in varying degree; and it's terminology is imprecise. Discussion about music, music theory and analysis, including its techniques, its description, critiques, and instructions to performers are very often described in words. Common musical notation is an attempt at precision, but even some elements of notation are vaguely defined. While words themselves have relative meaning, there is no absolute meaning of 'loud' or 'cantabile' or $<$ or $\boldsymbol{\imath}$. Fuzzy Logic has been formulated specifically to deal with the imprecision and 'vagueness' which is present when using natural language descriptors.

A significant feature of music is that the aesthetic outcome is often more than the sum of its technical elements. Indeed, what is the role of timbre, attack, duration, decay, articulation, spatialisation, register, texture, voicing, entries and timing, rhythm, tempo or meter? What does musical form, structure, or process contribute? In fact, it is often the means and details of the interactions between the distinct elements which significantly influence the effectiveness of the whole work. This means music is, technically, a non-linear system. "Non linear systems are systems that cannot be mathematically described as the sum of their components." This behaviour can be observed across a variety of disciplines including biology and economics as well as physics. [10]. Fuzzy Logic is good for modelling non-linear systems as little prior knowledge of the original system is required [7]. This is a significant reason for using Fuzzy Logic as the principle with which to build this theory of music.

It may be noted here that in practice musical features such as amplitude and register, flow in differing time scales. Hence my interest in pursuing granulation of time as a significant feature. The application of Fuzzy Logic through Granular Computing, which allows different attributes to be granulated in different sized chunks, will ultimately be a useful method [11][15.].

\section{Application of the Principles of Fuzzy Logic}

\subsection{Inputs to the Fuzzy Logic System}

One of the features of using Fuzzy Logic modelling is the possibility of using either expert or non-experts to provide inputs into the Fuzzy Logic system. These inputs include both identifying the elements which are significant to the situation, and the ways in which these elements interact with each other to produce outcomes.

Different types of systems have been built using expert and non-expert inputs. For example Fuzzy ART systems learn through repeated experience [3], while Fuzzy 
Logic inference engines, for example [8], operate on the basis of rules derived from the experience of experts in the field. At this point as a composer of many years standing and some academic achievement, I claim to be sufficiently expert to provide a provisional set of elements for this prototype model. This model will be refined over time using more complex works. Thus its development will, incorporate both input types.

\subsection{How Fuzzy Logic Works}

The most striking thing is the ability of Fuzzy Logic to contain imprecise, or 'vague' concepts. Here is a brief explanation of how Fuzzy Logic works with imprecision. The first relevant concept is that of the 'universe of discourse' for a musical attribute, for example 'register', which measures the relative pitch or frequency range of sound events. The universe of discourse for 'register' includes all possible registers. Within this universe of registers, there exist any number of sets applicable to 'register'. It should be noted here that the 'universe of discourse' does not have to be completely covered by defined sets in order for the logic to work.

However, each defined set contains elements which are closely related to each other. The sets are labelled with words. For examples some possible 'register' sets could be labelled, or speaking more technically, the attribute 'register' could be given a 'term set' of 'high', 'upper mid', 'lower mid', 'low', and 'deep'. Yet even within these registral locations there may still be ambiguity about what should be contained by these possible sets, for example 'upper mid' and 'upper'. In particular, the useful thing about fuzzy logic is its inclusivity. Variables do not have to take only binary (true/false) values, but everything can simultaneously be partially true.

Typical musical analysis would classify specific sound events, or even phrases, as being in only one set or another, however when using Fuzzy Logic can be classified in several or all categories.

Secondly, typical analytical decisions about how to classify a note, a chord, a sound, a section, or even a whole work are justified on the basis of the relations of some musical elements, while ignoring those that don't fit. The ambiguous harmonies in the Prelude to Tristan und Isolde, are a well known example. In instances like this, the useful thing about fuzzy logic is its inclusivity. Variables do not have to take only binary (true/false) values, but everything can simultaneously be partially true.

The basic proposition of Fuzzy Logic is that any object belongs to all possible sets, but with varying degrees of membership. Consequently, each object, in Fuzzy Logic, acquires several properties - the sets to which it is assigned, and also the 'degree' with which it is thought to belong to each set.

Recall that each universe of discourse refers to only one musical attribute, while the term sets refer to the range of values that specific attribute may take. For example, the attribute 'amplitude' may be given a 'term set' of three elements: Loud, Moderate and Soft.

The relevance of each elemental fuzzy set to each musical configuration is recorded through the allocation of membership values for that configuration to each of the elemental fuzzy sets. Membership values will be greater than zero only for those elemental sets which are relevant to a particular musical configuration. So, if the musical configuration does not seem to fit into a specific set to any degree, then the value 
of its membership for that set is zero. For example, it is possible that the register of a specific bar of music could be assigned to two sets: 'upper' with a membership value of 0.4 ; and 'upper-mid' with a membership value of 0.8 , which reflects my evaluation that at this point the music is more in the middle range than in the upper range, yet it does still contain some upper registral characteristics.

Although the actual membership values are determined on the basis of my expertise, rather than on some predetermined quantitative measure, there is a meta-rule at play here. Namely, that in places where a particular musical occurrence is ambiguous or 'vague' it will be classed in as many sets as it may possibly suit. One of the features of using Fuzzy Logic is that no features are lost, as the classification process allows for ambiguity, which in itself may be a significant feature of some music. Another aspect is that all details are maintained for future use, including those which which may yet prove to be significant, are not destroyed by early choices. It is my contention that expression in music is derived from the interplay of constituent elements over time.

Then, looking to the future purpose of this methodology, the feature of Fuzzy Logic which makes it practical and useable is the change in perspective from individual object to the fuzzy set as a whole. Since objects have been classified with a degree of membership of each set, then each set includes information regarding membership values of the objects within it. In this sense, each set then contains its own individual 'membership function' for its objects. These membership functions can then be used to represent each set in 'fuzzy' mathematics, according to strict logical principles applied through a mathematically precise and rigorous Fuzzy Logic inference engine. It is this procedure which will enable Fuzzy Logic to become a practical and useable control mechanism.

\subsection{Terminology}

The terminology I am developing has been generalised away from traditional analytical terms which originate from notational foundations. My terminology covers the chief characteristics by which sound itself is shaped and processed through compositional decision making. Terms, such as 'articulation: discrete', which describe the elemental details of the music, have been used to generalise characteristics across both acoustic and computer music domains. Thus the term 'sound events' is used instead of 'note' so that the palette of sounds available in computer music, which are likely to be unpitched, and a-rhythmic, can be accommodated. It is likely, however, that further refinement of both terminology and model will take place as more music is analysed using these principles.

The development of appropriate 'Term Sets' is a requirement of Fuzzy Logic, while the development of an appropriate connective 'Grammar' is an additional major implication of Granular Computing for the eventual Fuzzy Logic control mechanism.

\subsection{The Benefits of Fuzzy Logic Processing}

Granular Computing is a useful methodology because it can be used as an algorithmic compositional technique. It is a coherent conceptual and algorithmic method for the representation and processing of information [11]. Granular Computing, based on 
Fuzzy Logic techniques, provides the mechanism for the relatively new technique of 'computing with words' [15]. These methodologies are used to construct intelligent systems which can respond efficiently to variable contexts and data inputs. This has a number of benefits as the use of natural language grammar and linguistic variables, such as "deafening" rather than a numerical " $120 \mathrm{~dB}$ ", means that its logic is transparent and meaningful [4]. Although some elements of musical notation are vaguely defined, others are very specific, for example metronome markings . Granular Computing allows both vague and crisp elements to be combined.

Granulation is a process of compressing data by grouping objects because they may be indistinguishable, for example by masking; have similar properties; are closely located; or have similar functions. This clumping process may be crisp or fuzzy, physical or virtual, and may produce dense or sparse results. Fuzzy granulation is particularly useful in environments of imprecision, uncertainty and partial truth. This method permits the subjective aggregation of vague information. This leads to Granular Computing, where the data sets, or information granules, are labelled with words from natural language [23].

Time itself, is an important variable to be subjected to granulation [17]. This is immediately relevant to music which is a temporal art. Indeed, time is used in different ways in different elements of the music. Most musical attributes from small rhythmic details to larger formal structures, depend on various time scales.

Fuzzy Logic models can be used in a non-numerate way resulting in a methodology that is extremely user-friendly. Even though natural language descriptors may only provide ordinal information (that is the elements can be ranked in order, from lowest to highest preference) this may be enough for the problem to be solved [4]. While, if necessary, fuzzy sets can be developed from these descriptors, with membership functions, to provide a mathematical basis for calculating solutions.

\section{Future Work}

This proposal to use Fuzzy Logic to model and compose a musical work has already highlighted some important issues which may require detailed investigation in order to allow my research to progress to a useful and functional conclusion in the form of a compositional method.

These issues include the further refinement of a appropriate elemental sets and terminology, as well as their interaction through connective grammar, to allow implementation through a Fuzzy Logic control system.

Another issue to be addressed is the granulation of Time, since different musical elements change at different rates, thus different time scales are likely to be relevant. In fact a significant part of the work to be done, is to examine various musics to obtain some generalised time domain results, and to formulate terms for modelling different interactions between elements.

At this point my work is being conducted manually. Although ny present research will contribute to the development of digital tools, however, the first step is to identify the significant compositional elements. Once, these have been determined, then automated ways to compose music can be developed. 
The final issue is the development an appropriate perspective on a complete musical work as a self-determining system. The use of Fuzzy Logic implies that little prior knowledge of the whole system is required [7], however there may be other aspects of systems approach that could be usefully considered. There are models of control systems for terminating and non-terminating systems. Are these relevant to this work, now or in the future?

\section{Conclusion}

The requirements set out by Babbitt [1], that any musical theory be logical, coherent, useful, and intelligible are also met by Fuzzy Logic Principles. This is amplified when, as discussed earlier, Efstathiou's model of decision making is applied to the underlying question.

It is important that the modelling methodology allows for all these attributes while itself being rule bound, with evolutionary decision making, thus providing for variation in the elements and their combination over time. These are significant reasons for using Fuzzy Logic and Granular Computing as the principles on which to build this theory of music. Other reasons come from diverse quarters, together building a compelling argument for exploring this as a fruitful line of research. And finally, my initial investigations show that very little applied work has been done specifically in this area.

What distinguishes my work is the use of Fuzzy Logic principles, which allows for the incorporation of ambiguity, to assess at several levels, the construction of a broad range of Western music through two lenses simultaneously. Firstly, the detailed elemental perspective, that is the construction of the individual sound events in each piece of music. Secondly, the time domain perspective, that is how all these elements, and their variations, change and combine over time. Composers already do this kind of multidimensional thinking as an intrinsic part of their work. What differs from composer to composer and style to style, is the amount of, and which details in one or other of the dimensions they concern themselves with, the remainder being left to compositional algorithms.

Clearly, music is an excellent domain in which to apply Fuzzy Logic. In summary, a compositional method based on Fuzzy Logic principles can meet all these requirements, This is because each musical work can be conceptualised as a system, based on initial constraints and ongoing decisions made by the composer, between variables that are sometimes related and sometimes treated independently.

\section{References}

1. Babbitt, M.: The Structure and Function of Music Theory 1. College Music Symposium: Journal of the College Music Society 5, 49-60 (Fall 1965)

2. Cadiz, R.F.: Compositional Control of Computer Music by Fuzzy Logic, Ph.D. thesis, Northwestern University (2006)

3. Carpenter, G.A., Grossberg, S., et al.: Fuzzy ART: Fast Stable Learning and Categorisation of Analog Patterns by an Adaptive Resonance System. Neural Networks 4, 759-771 (1991a) 
4. Efstathiou, J.: Practical Multi-Attribute Decision Making and Fuzzy Set Theory. Studies in Management Sciences 20, 307-320 (1984)

5. Elsea, P.: Fuzzy Logic and Musical Decisions, ftp://arts.ucsc.edu/pub/ems/FUZzY/Fuzzy_logic_and_Music.pdf

6. Forte, A.: The Structure of Atonal Music. Yale University Press, New Haven (1973)

7. Kosko, B.: Fuzzy Thinking: The New Science of Fuzzy Logic. Flamingo, London (1994)

8. Mamdani, E.H., Ostergaard, J.J., et al.: Use of Fuzzy Logic for Implementing Rule Based Control of Industrial Processes. Studies in Management Sciences 20, 429-445 (1984)

9. Milicevic, M.: Positive Emotion Learning through Music Listening, http: / /myweb.lmu.edu/mmilicevic/NEWpers/_PAPERS/papers.html

10. Monash University's Complexity Virtual Lab, http://vlab.infotech.monash.edu.au/simulations/non-linear/

11. Pedrycz, W.: Granular Computing-The Emerging Paradigm. Journal of Uncertain Systems 1(1), 38-61 (2007)

12. Sorensen, A., Brown, A.: jMusic: Music Composition in Java, http://jmusic.ci.qut.edu.au/index.html

13. Suiter, W.: Text Manipulation: Voice with Audio or Acoustic Augmentation. MCA-Res thesis, Faculty of Creative Arts, University of Wollongong (2007)

14. Whalley, I.: Towards a Closed System Automated Composition Engine: Linking 'Kansei' and Musical Language Recombinicity. In: ICMC 2002, Goteborg, Sweden, pp. 200-203 (2002)

15. Zadeh, L.A.: Some reflections on soft computing, granular computing and their roles in the conception, design and utilization of information/intelligent systems. Soft Computing 2, 23-25 (1998) 